\title{
A semi-automated system for quantifying the oxidative potential of ambient particles in aqueous extracts using the dithiothreitol (DTT) assay: results from the Southeastern Center for Air Pollution and Epidemiology (SCAPE)
}

T. Fang et al.

Correspondence to: R. J. Weber (rodney.weber@eas.gatech.edu) 


\section{Supporting Information (SI)}

\section{Chemical preparation and storage information:}

PQN standard: $5 \mathrm{mM}$ 9,10-phenanthraquinone (PQN) standard stock was made by dissolving $10.4 \mathrm{mg}$ of 9,10-phenanthraquinone in $10 \mathrm{~mL}$ DMSO. The stock was kept in freezer when not in use. For routine analysis, final PQN standard was prepared from stock in deionized water (DI water, Nanopure Infinity ${ }^{\mathrm{TM}}$ ultrapure water system; $>18 \mathrm{M} \Omega \mathrm{cm}^{-1}$ ) before feeding to the system. 1 mM DTT: $10 \mathrm{mM}$ stock DTT solution was made by dissolving $154.24 \mathrm{mg}$ DTT in $100 \mathrm{~mL}$ DI water and stored in fridge no longer than one month. $1 \mathrm{mM}$ DTT was made fresh from 10mM stock solution every time for immediate use.

0.2 mM DTNB: $0.2 \mathrm{mM}$ DTNB was prepared in Methanol (MeOH), stored in fridge for no longer than 6 months.

0.08 M Tris buffer with 4 mM EDTA: Tris buffer was first prepared by dissolving $96.92 \mathrm{~g}$ of Tris Base and $11.69 \mathrm{~g}$ of EDTA in DI water. This will bring the $\mathrm{pH}$ to $\sim 10$. Then adjust the $\mathrm{pH}$ to 8.9 by adding small amount of $\mathrm{HCl}$. Add DI water to make a final volume of $10 \mathrm{~L}$ as the stock and store in room temperature.

0.5 M Potassium Phosphate Buffer (KBuffer): 0.5 M Kbuffer was made by mixing $13.47 \mathrm{~g}$ of $\mathrm{KH}_{2} \mathrm{PO}_{4}$ (Monopotassium Phosphate) and $69.85 \mathrm{~g}$ of $\mathrm{K}_{2} \mathrm{HPO}_{4}$ (Dipotassium Phosphate) in $1 \mathrm{~L}$ DI water. The mixture was then chelexed using Chelex ${ }^{\circledR} 100$ resin (BIO-RAD Laboratories, Inc., USA) to remove binding polyvalent metal ions (especially $\mathrm{Cu}$ and $\mathrm{Fe}$ ). $1 \% w / v T C A$ was prepared in DI water and stored in room temperature. 
Table S1. Comparison of semi-automated DTT assay system to the traditional manual method.

\begin{tabular}{|c|c|c|}
\hline & $\begin{array}{c}\text { Traditional manual } \\
\text { method }\end{array}$ & $\begin{array}{c}\text { Semi-automated } \\
\text { method }\end{array}$ \\
\hline Labor involved & Yes & No \\
\hline Numbers of samples analyzed daily & $5-8$ & 24 \\
\hline Time for each sample, $\mathrm{h}$ & 1 & 1 \\
\hline Remote control & No & Yes \\
\hline Reaction Volume, $\mathrm{mL}$ & 1 & 5 \\
\hline Reaction Vial & Multiple vials & Single vial \\
\hline Limit of Detection, nmol/min & $0.26(\mathrm{~N}=5)$ & $0.31(\mathrm{~N}=37)$ \\
\hline
\end{tabular}

Table S2. The seasonal variation on volume-normalized water-soluble DTT activity assessed by ANOVA tests

\begin{tabular}{|c|c|c|c|c|c|c|c|}
\hline Site & Season & \multicolumn{2}{|c|}{$\begin{array}{c}\text { Average } \pm \text { variance } \\
\left(\left(\mathrm{nmol} / \mathrm{min} / \mathrm{m}^{3}\right)\right.\end{array}$} & $\mathbf{N}$ & $\mathbf{F}$ & P-value & $F_{\text {critical }}$ \\
\hline \multirow{2}{*}{ YRK } & Summer & 0.28 & 0.01 & 33 & \multirow{2}{*}{0.01} & \multirow{2}{*}{0.91} & \multirow{2}{*}{7.14} \\
\hline & Winter & 0.28 & 0.01 & 22 & & & \\
\hline \multirow{3}{*}{ RS } & Fall & 0.33 & 0.01 & 29 & \multirow{3}{*}{3.87} & \multirow{3}{*}{0.03} & \multirow{3}{*}{4.89} \\
\hline & Winter & 0.36 & 0.02 & 31 & & & \\
\hline & Fall 2013 & 0.27 & 0.00 & 20 & & & \\
\hline \multirow{3}{*}{ GT } & Summer & 0.24 & 0.01 & 38 & \multirow{3}{*}{2.42} & \multirow{3}{*}{0.10} & \multirow{3}{*}{4.88} \\
\hline & Winter & 0.25 & 0.02 & 22 & & & \\
\hline & Fall 2013 & 0.20 & 0.00 & 23 & & & \\
\hline \multirow{6}{*}{ JST } & Summer & 0.29 & 0.02 & 31 & \multirow{6}{*}{3.15} & \multirow{6}{*}{0.01} & \multirow{6}{*}{3.13} \\
\hline & Summer & 0.29 & 0.00 & 37 & & & \\
\hline & Fall & 0.34 & 0.01 & 26 & & & \\
\hline & Winter & 0.43 & 0.03 & 22 & & & \\
\hline & Winter & 0.33 & 0.04 & 30 & & & \\
\hline & $\begin{array}{c}\text { Spring } \\
2013\end{array}$ & 0.32 & 0.03 & 22 & & & \\
\hline
\end{tabular}


Table S3. The seasonal variation on mass-normalized water-soluble DTT activity assessed by ANOVA tests

\begin{tabular}{|c|c|c|c|c|c|c|c|}
\hline Site & Season & \multicolumn{2}{|c|}{$\begin{array}{c}\text { Mean } \pm \text { variance } \\
(\mathrm{pmol} / \mathrm{min} / \mu \mathrm{g})\end{array}$} & $\mathbf{N}$ & $\mathbf{F}$ & P-value & $F_{\text {critical }}$ \\
\hline \multirow{2}{*}{ YRK } & Summer & 29.76 & 0.16 & 33 & \multirow{2}{*}{15.01} & \multirow{2}{*}{$3.01 \mathrm{E}-04$} & \multirow{2}{*}{7.15} \\
\hline & Winter & 42.38 & 0.10 & 21 & & & \\
\hline \multirow{3}{*}{$\mathrm{RS}$} & Fall & 31.15 & 0.02 & 29 & \multirow{3}{*}{1.84} & \multirow{3}{*}{0.17} & \multirow{3}{*}{3.12} \\
\hline & Winter & 35.65 & 0.07 & 28 & & & \\
\hline & Fall 2013 & 32.71 & 0.18 & 20 & & & \\
\hline \multirow{3}{*}{ GT } & Summer & 26.16 & 0.05 & 38 & \multirow{3}{*}{19.89} & \multirow{3}{*}{$1.05 \mathrm{E}-07$} & \multirow{3}{*}{3.11} \\
\hline & Winter & 30.60 & 0.06 & 20 & & & \\
\hline & Fall 2013 & 18.06 & 0.02 & 23 & & & \\
\hline \multirow{6}{*}{ JST } & Summer & 25.35 & 0.05 & 31 & \multirow{6}{*}{25.17} & \multirow{6}{*}{$9.33 \mathrm{E}-19$} & \multirow{6}{*}{3.13} \\
\hline & Summer & 29.86 & 0.03 & 37 & & & \\
\hline & Fall & 41.06 & 0.12 & 26 & & & \\
\hline & Winter & 55.54 & 0.28 & 22 & & & \\
\hline & Winter & 37.17 & 0.12 & 30 & & & \\
\hline & Winter & 36.82 & 0.15 & 23 & & & \\
\hline
\end{tabular}


(a)

- Sampling_Sites

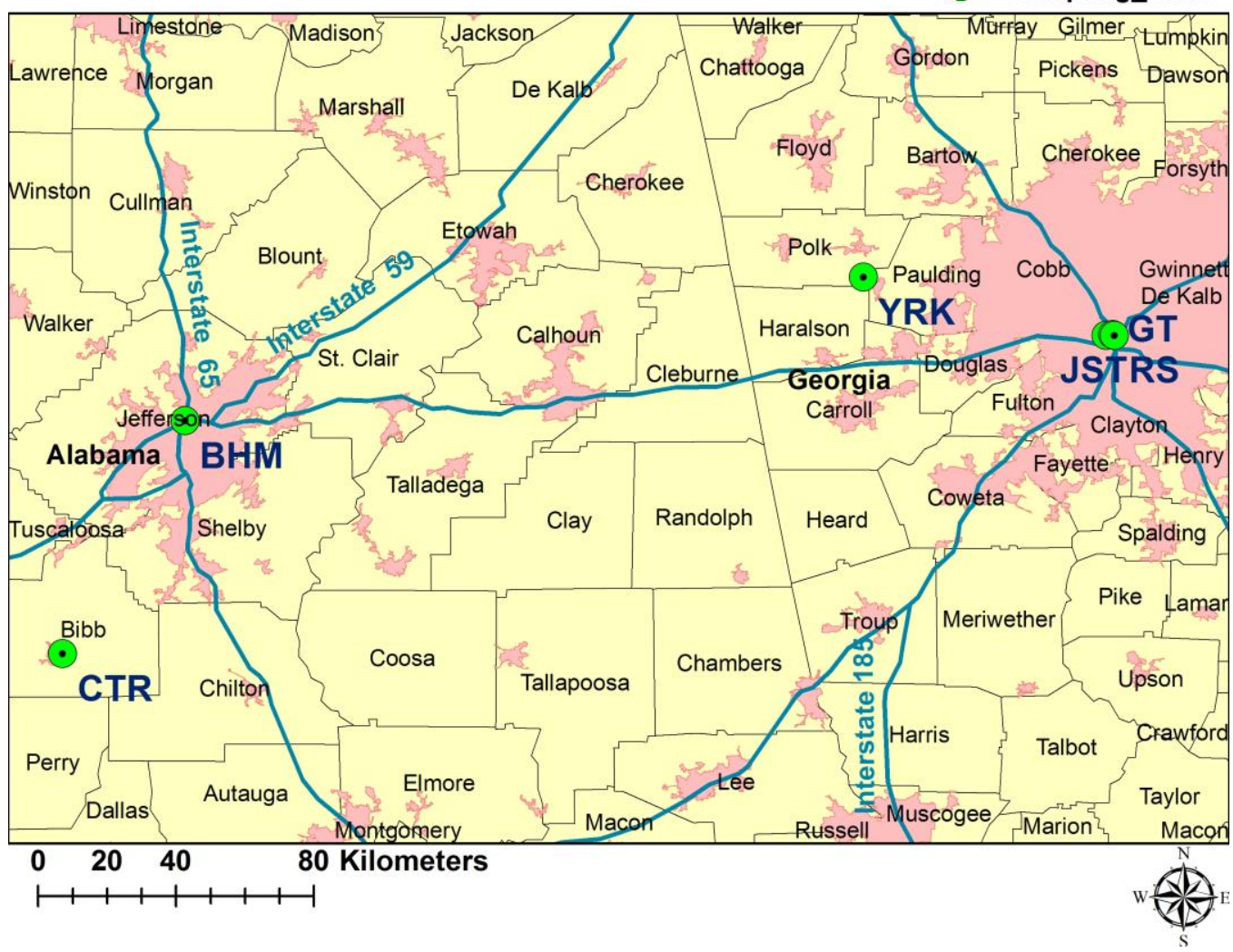

(b)

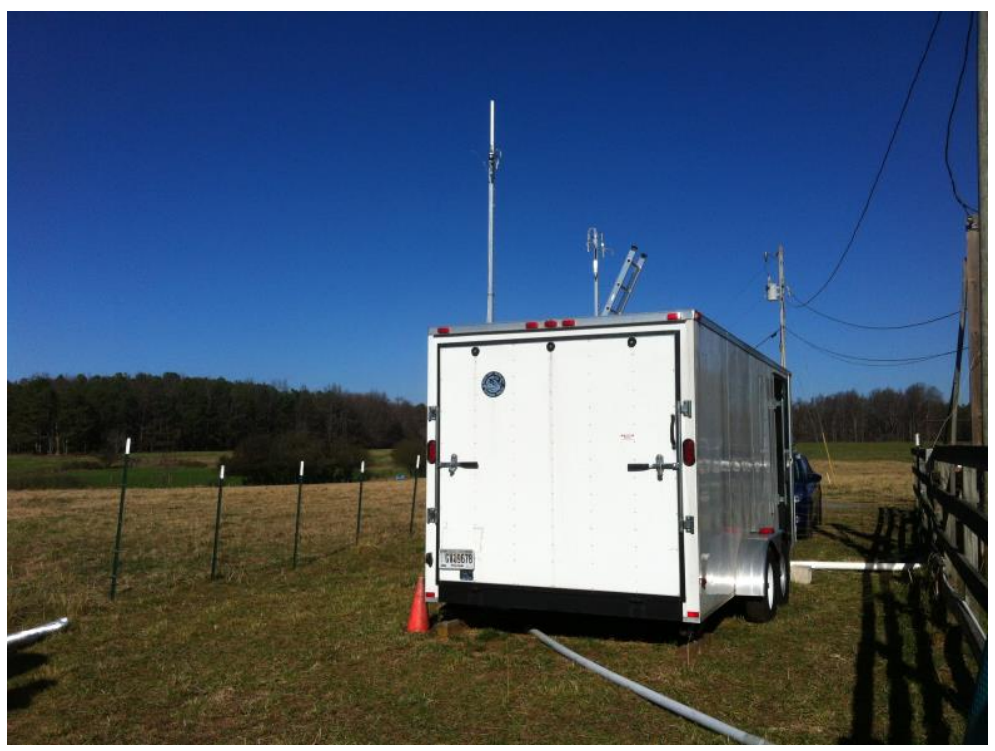

Figure S1. Map of sampling sites (a) and a picture of trailer at Yorkville (b) 


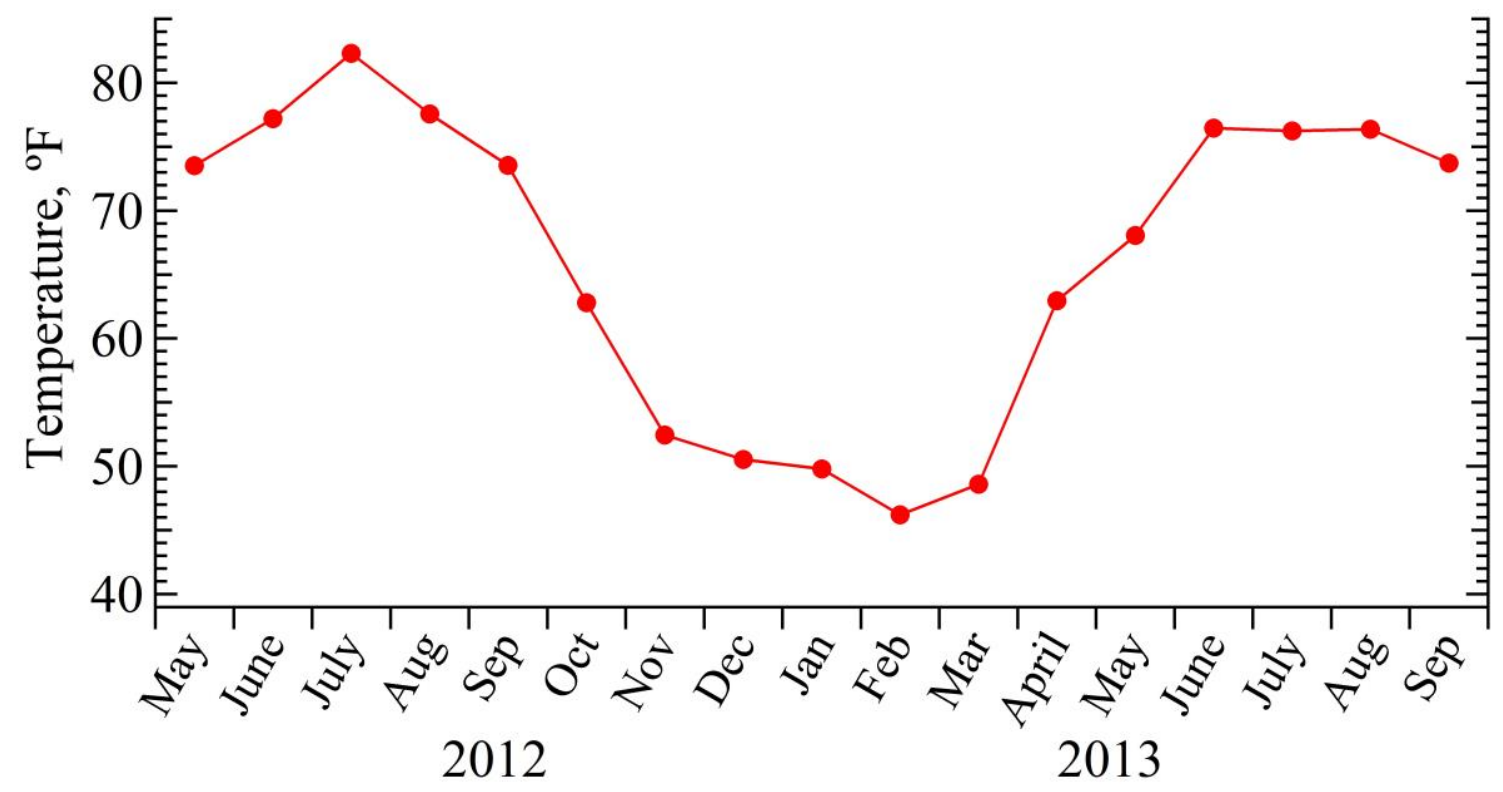

Figure S2. Temperature profile from May 2012 to September 2013 in Atlanta, GA.

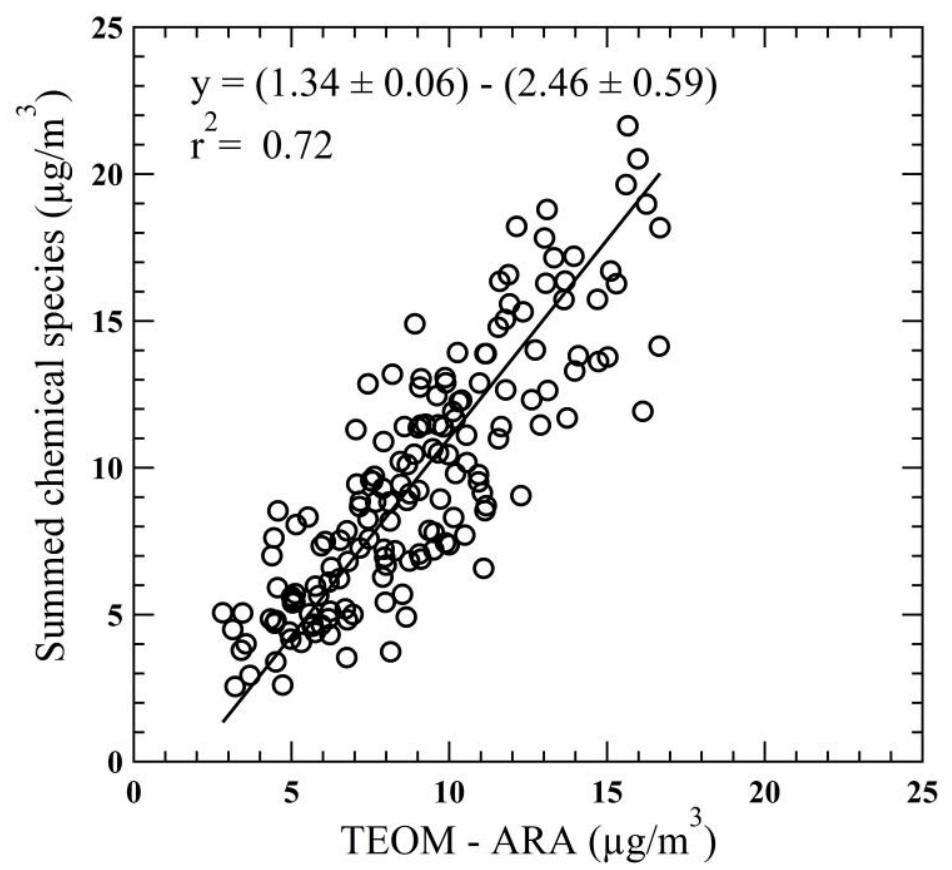


Figure S3. Comparison of estimated $\mathrm{PM}_{2.5}$ concentration from the sum of components determined from the High Volume filters, which included: EC, organic mass (OC*1.6), ammonium sulfate and water soluble metals concentration with the $\mathrm{PM}_{2.5}$ measured by TEOM from Atmospheric Research Analysis data archive at the SEARCH JST site. EC/OC - Sunset Laboratory TOT analyzer; water soluble metals - X-ray fluorescence instrument, XRF, Xact ${ }^{\mathrm{TM}}$ 625 Monitoring System [details would be presented in a subsequent publication (Fang et al.; in prep); $\left[\left(\mathrm{NH}_{4}\right)_{2} \mathrm{SO}_{4}\right]=\frac{M W_{\left(\mathrm{NH}_{4}\right)_{2} \mathrm{SO}_{4}}}{M W_{S}} \times[\mathrm{S}]$, assuming sulfate and ammonium are all $\left(\mathrm{NH}_{4}\right)_{2} \mathrm{SO}_{4}$ and $[\mathrm{S}]$ (sulfur) were obtained from XRF metals analysis. (The equation was obtained by orthogonal regression.) 
Hardware and software details of the Kloehn Control syringe pumps (Kloehn, Inc., Las

Vegas, Nevada, USA):

\section{Pump A:}

VersaPump 6, 48k resolution drive pump (P/N 55022);

12-way distribution rotary valve (P/N 24105);

$10 \mathrm{~mL}$ syringe (P/N 17600);

\section{Pump B:}

VersaPump 3, 12k resolution drive pump (P/N 23254);

6-way distribution valve (P/N 23327);

$250 \mu \mathrm{L}$ syringe (P/N 23166);

Software: Kloehn Control version 1.04

Kloehn Control program code for conducting the DTT method (Address 1 - pump A; Address 3 - pump B):

\begin{tabular}{|c|c|c|c|c|}
\hline Line & Line Label & Address & Command & Command Data \\
\hline 1 & & 1 & CONSTANT <varName $>=<$ float $>$ & dILarge, 12 \\
\hline 2 & & 1 & CONSTANT <varName $>=<$ float $>$ & AirLarge, 8 \\
\hline 3 & & 1 & CONSTANT < varName $>=<$ float $>$ & WasteLarge, 9 \\
\hline 4 & & 1 & CONSTANT <varName $>=<$ float $>$ & Kbuffer, 11 \\
\hline 5 & & 1 & CONSTANT $<$ varName $>=<$ float $>$ & Tris, 3 \\
\hline 6 & & 1 & CONSTANT <varName $>=<$ float $>$ & TCA, 4 \\
\hline 7 & & 1 & CONSTANT $<$ varName $>=<$ float $>$ & LWCC,5 \\
\hline 8 & & 1 & CONSTANT <varName $>=<$ float $>$ & Sample, 6 \\
\hline 9 & & 1 & CONSTANT < varName $>=<$ float $>$ & DTNB, 10 \\
\hline 10 & & 1 & CONSTANT <varName $>=<$ float $>$ & IVLarge, 1 \\
\hline 11 & & 1 & CONSTANT $<$ varName $>=<$ float $>$ & RVLarge, 2 \\
\hline 12 & & 3 & CONSTANT <varName $>=<$ float $>$ & dISmall,6 \\
\hline 13 & & 3 & CONSTANT < varName $>=<$ float $>$ & AirSmall,4 \\
\hline 14 & & 3 & CONSTANT $<$ varName $>=<$ float $>$ & WasteSmall,1 \\
\hline 15 & & 3 & CONSTANT $<$ varName $>=<$ float $>$ & DTT,5 \\
\hline
\end{tabular}




\begin{tabular}{|c|c|c|c|c|}
\hline 16 & & 3 & CONSTANT <varName > = <float $>$ & IVSmall,2 \\
\hline 17 & & 3 & CONSTANT <varName > = <float $>$ & RVSmall,3 \\
\hline \multicolumn{5}{|l|}{18} \\
\hline \multicolumn{5}{|l|}{19} \\
\hline 20 & HomePosition & 3 & SET out $2=\langle$ bit $>$ & 1 \\
\hline 21 & & 3 & SET out $2=\langle$ bit $>$ & 0 \\
\hline 22 & & 3 & SET out $2=\langle$ bit $>$ & 1 \\
\hline 23 & & 3 & SET out $1=\langle$ bit $>$ & 1 \\
\hline \multicolumn{5}{|l|}{24} \\
\hline 25 & PreloadTCA & 1 & VALVE_PORT $=\langle$ int $>[C C W]$ & TCA \\
\hline 26 & & 1 & $\begin{array}{l}\text { POSITION <float> [IMM or } \\
\text { SYNC] }\end{array}$ & 1000 \\
\hline 27 & & 1 & VALVE_PORT $=\langle$ int $\rangle[\mathrm{CCW}]$ & WasteLarge \\
\hline 28 & & 1 & $\begin{array}{l}\text { POSITION <float> [IMM or } \\
\text { SYNC] }\end{array}$ & 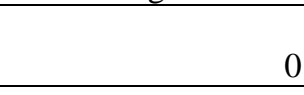 \\
\hline 29 & PreloadTris & 1 & VALVE_PORT $=\langle$ int $\rangle[\mathrm{CCW}]$ & Tris \\
\hline 30 & & 1 & $\begin{array}{l}\text { POSITION <float> [IMM or } \\
\text { SYNC] }\end{array}$ & 1000 \\
\hline 31 & & 1 & VALVE_PORT $=\langle$ int $>[\mathrm{CCW}]$ & WasteLarge \\
\hline 32 & & 1 & $\begin{array}{l}\text { POSITION <float> [IMM or } \\
\text { SYNC] }\end{array}$ & 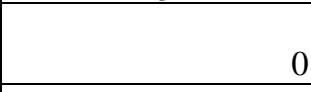 \\
\hline 33 & PreloadKbuffer & 1 & VALVE_PORT $=\langle$ int $\rangle[\mathrm{CCW}]$ & Kbuffer \\
\hline 34 & & 1 & $\begin{array}{l}\text { POSITION <float> [IMM or } \\
\text { SYNC] }\end{array}$ & 1000 \\
\hline 35 & & 1 & VALVE_PORT $=\langle$ int $\rangle[\mathrm{CCW}]$ & WasteLarge \\
\hline 36 & & 1 & $\begin{array}{l}\text { POSITION <float> [IMM or } \\
\text { SYNC] }\end{array}$ & 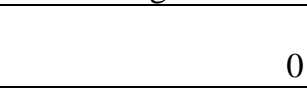 \\
\hline 37 & & 1 & VALVE_PORT $=\langle$ int $\rangle[\mathrm{CCW}]$ & dILarge \\
\hline 38 & & 1 & $\begin{array}{l}\text { POSITION <float> [IMM or } \\
\text { SYNC] }\end{array}$ & 5000 \\
\hline 39 & & 1 & VALVE_PORT $=\langle$ int $\rangle[\mathrm{CCW}]$ & WasteLarge \\
\hline 40 & & 1 & $\begin{array}{l}\text { POSITION <float> [IMM or } \\
\text { SYNC] }\end{array}$ & 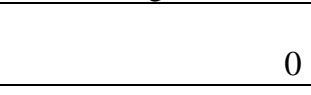 \\
\hline \multicolumn{5}{|l|}{41} \\
\hline 42 & PreloadDTNB & 1 & VALVE_PORT $=\langle$ int $>[\mathrm{CCW}]$ & DTNB \\
\hline 43 & & 1 & $\begin{array}{l}\text { POSITION <float> [IMM or } \\
\text { SYNC] }\end{array}$ & 1000 \\
\hline 44 & & 1 & VALVE_PORT $=\langle$ int $\rangle[\mathrm{CCW}]$ & WasteLarge \\
\hline 45 & & 1 & $\begin{array}{l}\text { POSITION <float> [IMM or } \\
\text { SYNC] }\end{array}$ & 0 \\
\hline 46 & PreloadDTT & 3 & VALVE_PORT $=\langle$ int $>[\mathrm{CCW}]$ & DTT \\
\hline 47 & & 3 & SET speed $=<$ float $>$ & 30 \\
\hline 48 & & 3 & $\begin{array}{l}\text { POSITION <float> [IMM or } \\
\text { SYNC] }\end{array}$ & 250 \\
\hline 49 & & 3 & VALVE_PORT $=\langle$ int $>[C C W]$ & WasteSmall \\
\hline 50 & & 3 & $\begin{array}{l}\text { POSITION <float> [IMM or } \\
\text { SYNC] }\end{array}$ & 0 \\
\hline
\end{tabular}




\begin{tabular}{|c|c|c|c|c|}
\hline \multicolumn{5}{|l|}{51} \\
\hline 52 & & & DO & \\
\hline 53 & ChangeSample & 3 & SET out $1=\langle$ bit $>$ & 0 \\
\hline 54 & & 3 & SET out $1=\langle$ bit $>$ & 1 \\
\hline \multicolumn{5}{|l|}{55} \\
\hline \multicolumn{5}{|l|}{56} \\
\hline 57 & PreloadSample & 1 & VALVE_PORT $=\langle$ int $>[C C W]$ & Sample \\
\hline 58 & & 1 & SET speed $=\langle$ float $\rangle$ & 400 \\
\hline 59 & & 1 & $\begin{array}{l}\text { POSITION <float> [IMM or } \\
\text { SYNC] }\end{array}$ & 1000 \\
\hline 60 & & 1 & VALVE_PORT $=\langle$ int $>[\mathrm{CCW}]$ & WasteLarge \\
\hline 61 & & 1 & $\begin{array}{l}\text { POSITION <float> [IMM or } \\
\text { SYNC] }\end{array}$ & 0 \\
\hline 62 & & 1 & DELAY <float $>$ & 1 \\
\hline \multicolumn{5}{|l|}{63} \\
\hline 64 & CleanLarge+Small & & DO & \\
\hline 65 & & 1 & VALVE_PORT $=\langle$ int $>[C C W]$ & dILarge \\
\hline 66 & & 1 & $\begin{array}{l}\text { POSITION <float> [IMM or } \\
\text { SYNC] }\end{array}$ & 5000 \\
\hline 67 & & 1 & VALVE_PORT $=\langle$ int $\rangle[\mathrm{CCW}]$ & WasteLarge \\
\hline 68 & & 1 & $\begin{array}{l}\text { POSITION <float> [IMM or } \\
\text { SYNC] }\end{array}$ & 0 \\
\hline 69 & & 3 & VALVE_PORT $=\langle$ int $>[\mathrm{CCW}]$ & dISmall \\
\hline 70 & & 3 & $\begin{array}{l}\text { POSITION <float> [IMM or } \\
\text { SYNC] }\end{array}$ & 250 \\
\hline 71 & & 3 & VALVE_PORT $=\langle$ int $>[\mathrm{CCW}]$ & WasteSmall \\
\hline 72 & & 3 & $\begin{array}{l}\text { POSITION <float> [IMM or } \\
\text { SYNC] }\end{array}$ & 0 \\
\hline 73 & & & LOOP <int $>$ & 3 \\
\hline \multicolumn{5}{|l|}{74} \\
\hline 75 & LoadReactionVial & 1 & VALVE_PORT $=\langle$ int $\rangle[\mathrm{CCW}]$ & Kbuffer \\
\hline 76 & & 1 & $\begin{array}{l}\text { POSITION <float> [IMM or } \\
\text { SYNC] }\end{array}$ & 1000 \\
\hline 77 & & 1 & VALVE_PORT $=\langle$ int $>[\mathrm{CCW}]$ & RVLarge \\
\hline 78 & & 1 & $\begin{array}{l}\text { POSITION < float> [IMM or } \\
\text { SYNC] }\end{array}$ & 0 \\
\hline 79 & & 1 & VALVE_PORT $=\langle$ int $>[C C W]$ & Sample \\
\hline 80 & & 1 & $\begin{array}{l}\text { POSITION <float> [IMM or } \\
\text { SYNC] }\end{array}$ & 3500 \\
\hline 81 & & 1 & DELAY <float> & 1 \\
\hline 82 & & 1 & VALVE_PORT $=\langle$ int $>[C C W]$ & RVLarge \\
\hline 83 & & 1 & $\begin{array}{l}\text { POSITION <float> [IMM or } \\
\text { SYNC] }\end{array}$ & 0 \\
\hline 84 & & 1 & VALVE_PORT $=\langle$ int $\rangle[\mathrm{CCW}]$ & AirLarge \\
\hline 85 & & 1 & $\begin{array}{l}\text { POSITION <float> [IMM or } \\
\text { SYNC] }\end{array}$ & 2000 \\
\hline 86 & & 1 & VALVE_PORT $=\langle$ int $\rangle[\mathrm{CCW}]$ & RVLarge \\
\hline
\end{tabular}




\begin{tabular}{|c|c|c|c|c|}
\hline 87 & & 1 & $\begin{array}{l}\text { POSITION < float> [IMM or } \\
\text { SYNC] }\end{array}$ & 0 \\
\hline 88 & & 1 & VALVE_PORT $=\langle$ int $\rangle[\mathrm{CCW}]$ & dILarge \\
\hline 89 & & 1 & $\begin{array}{l}\text { POSITION <float> [IMM or } \\
\text { SYNC] }\end{array}$ & 5000 \\
\hline 90 & & 1 & VALVE_PORT $=\langle$ int $>[\mathrm{CCW}]$ & WasteLarge \\
\hline 91 & & 1 & $\begin{array}{l}\text { POSITION <float> [IMM or } \\
\text { SYNC] }\end{array}$ & 0 \\
\hline 92 & & 3 & VALVE_PORT $=\langle$ int $\rangle[\mathrm{CCW}]$ & DTT \\
\hline 93 & & 3 & $\begin{array}{l}\text { POSITION <float> [IMM or } \\
\text { SYNC] }\end{array}$ & 250 \\
\hline 94 & & 3 & VALVE_PORT $=\langle$ int $>[\mathrm{CCW}]$ & RVSmall \\
\hline 95 & & 3 & SET speed $=\langle$ float $>$ & 40 \\
\hline 96 & & 3 & $\begin{array}{l}\text { POSITION <float> [IMM or } \\
\text { SYNC] }\end{array}$ & 0 \\
\hline 97 & & 3 & VALVE_PORT $=\langle$ int $\rangle[\mathrm{CCW}]$ & DTT \\
\hline 98 & & 3 & SET speed $=\langle$ float $>$ & 30 \\
\hline 99 & & 3 & $\begin{array}{l}\text { POSITION <float> [IMM or } \\
\text { SYNC] }\end{array}$ & 250 \\
\hline 100 & & 3 & VALVE_PORT $=\langle$ int $\rangle[\mathrm{CCW}]$ & RVSmall \\
\hline 101 & & 3 & SET speed $=\langle$ float $>$ & 40 \\
\hline 102 & & 3 & $\begin{array}{l}\text { POSITION <float> [IMM or } \\
\text { SYNC] }\end{array}$ & 0 \\
\hline 103 & & 3 & VALVE_PORT $=\langle$ int $>[\mathrm{CCW}]$ & AirSmall \\
\hline 104 & & 3 & $\begin{array}{l}\text { POSITION <float> [IMM or } \\
\text { SYNC] }\end{array}$ & 250 \\
\hline 105 & & 3 & VALVE_PORT $=\langle$ int $>[\mathrm{CCW}]$ & RVSmall \\
\hline 106 & & 3 & $\begin{array}{l}\text { POSITION <float> [IMM or } \\
\text { SYNC] }\end{array}$ & 0 \\
\hline 107 & & 1 & VALVE_PORT $=\langle$ int $\rangle[\mathrm{CCW}]$ & AirLarge \\
\hline 108 & & 1 & $\begin{array}{l}\text { POSITION <float> [IMM or } \\
\text { SYNC] }\end{array}$ & 5000 \\
\hline 109 & & 1 & VALVE_PORT $=\langle$ int $>[\mathrm{CCW}]$ & RVLarge \\
\hline 110 & & 1 & SET speed $=\langle$ float $>$ & 1000 \\
\hline 111 & & 1 & $\begin{array}{l}\text { POSITION <float> [IMM or } \\
\text { SYNC] }\end{array}$ & 0 \\
\hline 112 & & & DO & \\
\hline 113 & & 3 & VALVE_PORT $=\langle$ int $>[\mathrm{CCW}]$ & dISmall \\
\hline 114 & & 3 & SET speed $=\langle$ float $>$ & 30 \\
\hline 115 & & 3 & $\begin{array}{l}\text { POSITION <float> [IMM or } \\
\text { SYNC] }\end{array}$ & 250 \\
\hline 116 & & 3 & VALVE_PORT $=\langle$ int $\rangle[\mathrm{CCW}]$ & WasteSmall \\
\hline 117 & & 3 & $\begin{array}{l}\text { POSITION <float> [IMM or } \\
\text { SYNC] }\end{array}$ & 0 \\
\hline 118 & & & LOOP <int> & 3 \\
\hline 119 & & & & \\
\hline 120 & & & DO & \\
\hline 121 & LoadTCA & 1 & VALVE_PORT $=\langle$ int $\rangle[\mathrm{CCW}]$ & TCA \\
\hline
\end{tabular}




\begin{tabular}{|c|c|c|c|c|}
\hline 122 & & 1 & SET speed = <float $>$ & 400 \\
\hline 123 & & 1 & $\begin{array}{l}\text { POSITION < float> [IMM or } \\
\text { SYNC] }\end{array}$ & 1000 \\
\hline 124 & & 1 & VALVE_PORT $=\langle$ int $>[\mathrm{CCW}]$ & IVLarge \\
\hline 125 & & 1 & $\begin{array}{l}\text { POSITION <float> [IMM or } \\
\text { SYNC] }\end{array}$ & 0 \\
\hline 126 & & 1 & VALVE_PORT $=\langle$ int $>[C C W]$ & AirLarge \\
\hline 127 & & 1 & $\begin{array}{l}\text { POSITION < float> [IMM or } \\
\text { SYNC] }\end{array}$ & 1000 \\
\hline 128 & & 1 & VALVE_PORT $=\langle$ int $>[C C W]$ & IVLarge \\
\hline 129 & & 1 & $\begin{array}{l}\text { POSITION <float> [IMM or } \\
\text { SYNC] }\end{array}$ & 0 \\
\hline 130 & & 1 & VALVE_PORT $=\langle$ int $>[C C W]$ & dILarge \\
\hline 131 & & 1 & $\begin{array}{l}\text { POSITION < float> [IMM or } \\
\text { SYNC] }\end{array}$ & 1500 \\
\hline 132 & & 1 & VALVE_PORT $=\langle$ int $>[C C W]$ & WasteLarge \\
\hline 133 & & 1 & $\begin{array}{l}\text { POSITION < float> [IMM or } \\
\text { SYNC] }\end{array}$ & 0 \\
\hline 134 & & & & \\
\hline 135 & CleanLWCC & 1 & VALVE_PORT $=\langle$ int $>[C C W]$ & dILarge \\
\hline 136 & & 1 & $\begin{array}{l}\text { POSITION < float> [IMM or } \\
\text { SYNC] }\end{array}$ & 5000 \\
\hline 137 & & 1 & VALVE_PORT $=\langle$ int $>[C C W]$ & LWCC \\
\hline 138 & & 1 & $\begin{array}{l}\text { POSITION <float> [IMM or } \\
\text { SYNC] }\end{array}$ & 0 \\
\hline 139 & & & & \\
\hline 140 & Draw & 3 & VALVE_PORT $=\langle$ int $\rangle[\mathrm{CCW}]$ & RVSmall \\
\hline 141 & & 3 & $\begin{array}{l}\text { POSITION < float> [IMM or } \\
\text { SYNC] }\end{array}$ & 250 \\
\hline 142 & & 3 & VALVE_PORT $=\langle$ int $>[\mathrm{CCW}]$ & WasteSmall \\
\hline 143 & & 3 & $\begin{array}{l}\text { POSITION <float> [IMM or } \\
\text { SYNC] }\end{array}$ & 0 \\
\hline 144 & & 3 & VALVE_PORT $=\langle$ int $>[C C W]$ & RVSmall \\
\hline 145 & & 3 & $\begin{array}{l}\text { POSITION < float> [IMM or } \\
\text { SYNC] }\end{array}$ & 250 \\
\hline 146 & & 3 & VALVE_PORT $=\langle$ int $>[\mathrm{CCW}]$ & WasteSmall \\
\hline 147 & & 3 & $\begin{array}{l}\text { POSITION <float> [IMM or } \\
\text { SYNC] }\end{array}$ & 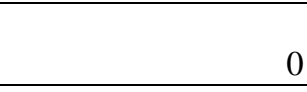 \\
\hline 148 & & 3 & VALVE_PORT $=\langle$ int $>[\mathrm{CCW}]$ & RVSmall \\
\hline 149 & & 3 & $\begin{array}{l}\text { POSITION <float> [IMM or } \\
\text { SYNC] }\end{array}$ & 250 \\
\hline 150 & & 3 & VALVE_PORT $=\langle$ int $>[\mathrm{CCW}]$ & WasteSmall \\
\hline 151 & & 3 & $\begin{array}{l}\text { POSITION <float> [IMM or } \\
\text { SYNC] }\end{array}$ & 180 \\
\hline 152 & & 3 & VALVE_PORT $=\langle$ int $>[\mathrm{CCW}]$ & IVSmall \\
\hline 153 & & 3 & $\begin{array}{l}\text { POSITION <float> [IMM or } \\
\text { SYNC] }\end{array}$ & 80 \\
\hline 154 & & 3 & VALVE_PORT $=\langle$ int $>[\mathrm{CCW}]$ & RVSmall \\
\hline 155 & & 3 & POSITION < float> [IMM or & 0 \\
\hline
\end{tabular}




\begin{tabular}{|c|c|c|c|c|}
\hline & & & SYNC] & \\
\hline 156 & & 3 & VALVE_PORT $=\langle$ int $>[\mathrm{CCW}]$ & AirSmall \\
\hline 157 & & 3 & $\begin{array}{l}\text { POSITION <float> [IMM or } \\
\text { SYNC] }\end{array}$ & 250 \\
\hline 158 & & 3 & VALVE_PORT $=\langle$ int $>[C C W]$ & RVSmall \\
\hline 159 & & 3 & $\begin{array}{l}\text { POSITION <float> [IMM or } \\
\text { SYNC] }\end{array}$ & 0 \\
\hline 160 & & 3 & VALVE_PORT $=\langle$ int $>[C C W]$ & AirSmall \\
\hline 161 & & 3 & $\begin{array}{l}\text { POSITION <float> [IMM or } \\
\text { SYNC] }\end{array}$ & 250 \\
\hline 162 & & 3 & VALVE_PORT $=\langle$ int $>[C C W]$ & IVSmall \\
\hline 163 & & 3 & $\begin{array}{l}\text { POSITION <float> [IMM or } \\
\text { SYNC] }\end{array}$ & 0 \\
\hline \multicolumn{5}{|l|}{164} \\
\hline 165 & LoadTris & 1 & VALVE_PORT $=\langle$ int $>[C C W]$ & Tris \\
\hline 166 & & 1 & $\begin{array}{l}\text { POSITION <float> }[\text { IMM or } \\
\text { SYNC] }\end{array}$ & 2000 \\
\hline 167 & & 1 & VALVE_PORT $=\langle$ int $>[\mathrm{CCW}]$ & IVLarge \\
\hline 168 & & 1 & $\begin{array}{l}\text { POSITION <float> [IMM or } \\
\text { SYNC] }\end{array}$ & 0 \\
\hline 169 & & 1 & VALVE_PORT $=\langle$ int $>[\mathrm{CCW}]$ & AirLarge \\
\hline 170 & & 1 & $\begin{array}{l}\text { POSITION <float> [IMM or } \\
\text { SYNC] }\end{array}$ & 1000 \\
\hline 171 & & 1 & VALVE_PORT $=\langle$ int $>[\mathrm{CCW}]$ & IVLarge \\
\hline 172 & & 1 & $\begin{array}{l}\text { POSITION <float> [IMM or } \\
\text { SYNC] }\end{array}$ & 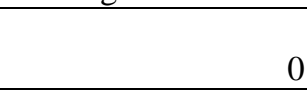 \\
\hline 173 & & 1 & VALVE_PORT $=\langle$ int $>[\mathrm{CCW}]$ & dILarge \\
\hline 174 & & 1 & $\begin{array}{l}\text { POSITION <float> [IMM or } \\
\text { SYNC] }\end{array}$ & 5000 \\
\hline 175 & & 1 & VALVE_PORT $=\langle$ int $>[\mathrm{CCW}]$ & WasteLarge \\
\hline 176 & & 1 & $\begin{array}{l}\text { POSITION <float> [IMM or } \\
\text { SYNC] }\end{array}$ & 0 \\
\hline \multicolumn{5}{|l|}{177} \\
\hline 178 & LoadDTNB & 1 & VALVE_PORT $=\langle$ int $>[C C W]$ & DTNB \\
\hline 179 & & 1 & $\begin{array}{l}\text { POSITION < float> [IMM or } \\
\text { SYNC] }\end{array}$ & 500 \\
\hline 180 & & 1 & VALVE_PORT $=\langle$ int $>[\mathrm{CCW}]$ & IVLarge \\
\hline 181 & & 1 & $\begin{array}{l}\text { POSITION <float> [IMM or } \\
\text { SYNC] }\end{array}$ & 0 \\
\hline 182 & & 1 & VALVE_PORT $=\langle$ int $>[\mathrm{CCW}]$ & dILarge \\
\hline 183 & & 1 & $\begin{array}{l}\text { POSITION < float> [IMM or } \\
\text { SYNC] }\end{array}$ & 1800 \\
\hline 184 & & 1 & VALVE_PORT $=\langle$ int $>[\mathrm{CCW}]$ & IVLarge \\
\hline 185 & & 1 & $\begin{array}{l}\text { POSITION <float }>\text { [IMM or } \\
\text { SYNC] }\end{array}$ & 0 \\
\hline 186 & & 1 & VALVE_PORT $=\langle$ int $\rangle[\mathrm{CCW}]$ & IVLarge \\
\hline 187 & & 1 & $\begin{array}{l}\text { POSITION < float> [IMM or } \\
\text { SYNC] }\end{array}$ & 5000 \\
\hline 188 & & 1 & VALVE_PORT $=\langle$ int $>[\mathrm{CCW}]$ & IVLarge \\
\hline
\end{tabular}




\begin{tabular}{|c|c|c|c|c|}
\hline 189 & & 1 & $\begin{array}{l}\text { POSITION < float> [IMM or } \\
\text { SYNC] }\end{array}$ & 0 \\
\hline 190 & & 1 & VALVE_PORT $=\langle$ int $>[\mathrm{CCW}]$ & AirLarge \\
\hline 191 & & 1 & $\begin{array}{l}\text { POSITION <float> [IMM or } \\
\text { SYNC] }\end{array}$ & 5000 \\
\hline 192 & & 1 & VALVE $P O R T=\langle$ int $>[C C W]$ & IVLarge \\
\hline 193 & & 1 & $\begin{array}{l}\text { POSITION <float> [IMM or } \\
\text { SYNC] }\end{array}$ & 0 \\
\hline \multicolumn{5}{|l|}{194} \\
\hline 195 & IVtoLWCC & 1 & VALVE_PORT $=\langle$ int $\rangle[\mathrm{CCW}]$ & IVLarge \\
\hline 196 & & 1 & $\begin{array}{l}\text { POSITION <float> [IMM or } \\
\text { SYNC] }\end{array}$ & 4700 \\
\hline 197 & & 1 & VALVE_PORT $=\langle$ int $\rangle[\mathrm{CCW}]$ & WasteLarge \\
\hline 198 & & 1 & $\begin{array}{l}\text { POSITION <float> [IMM or } \\
\text { SYNC] }\end{array}$ & 4000 \\
\hline 199 & & 1 & VALVE_PORT $=\langle$ int $>[\mathrm{CCW}]$ & LWCC \\
\hline 200 & & 1 & SET speed $=<$ float $>$ & 400 \\
\hline 201 & & 1 & $\begin{array}{l}\text { POSITION <float> [IMM or } \\
\text { SYNC] }\end{array}$ & 0 \\
\hline \multicolumn{5}{|l|}{202} \\
\hline 203 & RinseIV & 1 & VALVE_PORT $=\langle$ int $>[\mathrm{CCW}]$ & IVLarge \\
\hline 204 & & 1 & SET speed $=<$ float $>$ & 400 \\
\hline 205 & & 1 & $\begin{array}{l}\text { POSITION < float> [IMM or } \\
\text { SYNC] }\end{array}$ & 2000 \\
\hline 206 & & 1 & VALVE_PORT $=\langle$ int $\rangle[\mathrm{CCW}]$ & WasteLarge \\
\hline 207 & & 1 & $\begin{array}{l}\text { POSITION <float> [IMM or } \\
\text { SYNC] }\end{array}$ & 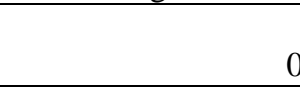 \\
\hline 208 & & & DO & \\
\hline 209 & & 1 & VALVE_PORT $=\langle$ int $>[\mathrm{CCW}]$ & dILarge \\
\hline 210 & & 1 & $\begin{array}{l}\text { POSITION <float> [IMM or } \\
\text { SYNC] }\end{array}$ & 5000 \\
\hline 211 & & 1 & VALVE_PORT $=\langle$ int $>[\mathrm{CCW}]$ & IVLarge \\
\hline 212 & & 1 & $\begin{array}{l}\text { POSITION <float> [IMM or } \\
\text { SYNC] }\end{array}$ & 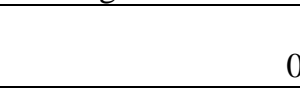 \\
\hline 213 & & 1 & VALVE_PORT $=\langle$ int $>[\mathrm{CCW}]$ & dILarge \\
\hline 214 & & 1 & $\begin{array}{l}\text { POSITION < float> [IMM or } \\
\text { SYNC] }\end{array}$ & 3000 \\
\hline 215 & & 1 & VALVE_PORT $=\langle$ int $\rangle[\mathrm{CCW}]$ & IVLarge \\
\hline 216 & & 1 & $\begin{array}{l}\text { POSITION <float> [IMM or } \\
\text { SYNC] }\end{array}$ & 0 \\
\hline 217 & & 1 & VALVE_PORT $=\langle$ int $\rangle[\mathrm{CCW}]$ & IVLarge \\
\hline 218 & & 1 & $\begin{array}{l}\text { POSITION <float> [IMM or } \\
\text { SYNC] }\end{array}$ & 5000 \\
\hline 219 & & 1 & VALVE_PORT $=\langle$ int $>[\mathrm{CCW}]$ & WasteLarge \\
\hline 220 & & 1 & $\begin{array}{l}\text { POSITION <float> [IMM or } \\
\text { SYNC] }\end{array}$ & 0 \\
\hline 221 & & 1 & VALVE_PORT $=\langle$ int $>[\mathrm{CCW}]$ & IVLarge \\
\hline 222 & & 1 & $\begin{array}{l}\text { POSITION <float> [IMM or } \\
\text { SYNC] }\end{array}$ & 5000 \\
\hline
\end{tabular}




\begin{tabular}{|c|c|c|c|c|}
\hline 223 & & 1 & VALVE_PORT $=\langle$ int $\rangle[\mathrm{CCW}]$ & WasteLarge \\
\hline 224 & & 1 & $\begin{array}{l}\text { POSITION < float> [IMM or } \\
\text { SYNC] }\end{array}$ & 0 \\
\hline 225 & & & LOOP <int $>$ & 2 \\
\hline 226 & & & LOOP <int> & 5 \\
\hline \multicolumn{5}{|l|}{227} \\
\hline 228 & RinseRV & 1 & VALVE_PORT $=\langle$ int $\rangle[\mathrm{CCW}]$ & RVLarge \\
\hline 229 & & 1 & $\begin{array}{l}\text { POSITION <float> [IMM or } \\
\text { SYNC] }\end{array}$ & 5000 \\
\hline 230 & & 1 & VALVE_PORT $=\langle$ int $\rangle[\mathrm{CCW}]$ & WasteLarge \\
\hline 231 & & 1 & $\begin{array}{l}\text { POSITION <float> [IMM or } \\
\text { SYNC] }\end{array}$ & 0 \\
\hline 232 & & & DO & \\
\hline 233 & & 1 & VALVE_PORT $=\langle$ int $\rangle[\mathrm{CCW}]$ & dILarge \\
\hline 234 & & 1 & $\begin{array}{l}\text { POSITION <float> [IMM or } \\
\text { SYNC] }\end{array}$ & 5000 \\
\hline 235 & & 1 & VALVE_PORT $=\langle$ int $\rangle[\mathrm{CCW}]$ & RVLarge \\
\hline 236 & & 1 & $\begin{array}{l}\text { POSITION <float> [IMM or } \\
\text { SYNC] }\end{array}$ & 0 \\
\hline 237 & & 1 & VALVE_PORT $=\langle$ int $\rangle[\mathrm{CCW}]$ & dILarge \\
\hline 238 & & 1 & $\begin{array}{l}\text { POSITION <float> [IMM or } \\
\text { SYNC] }\end{array}$ & 3000 \\
\hline 239 & & 1 & VALVE_PORT $=\langle$ int $\rangle[\mathrm{CCW}]$ & RVLarge \\
\hline 240 & & 1 & $\begin{array}{l}\text { POSITION <float> [IMM or } \\
\text { SYNC] }\end{array}$ & 0 \\
\hline 241 & & 1 & VALVE_PORT $=\langle$ int $>[\mathrm{CCW}]$ & RVLarge \\
\hline 242 & & 1 & $\begin{array}{l}\text { POSITION <float> [IMM or } \\
\text { SYNC] }\end{array}$ & 5000 \\
\hline 243 & & 1 & VALVE_PORT $=\langle$ int $\rangle[\mathrm{CCW}]$ & WasteLarge \\
\hline 244 & & 1 & $\begin{array}{l}\text { POSITION <float> [IMM or } \\
\text { SYNC] }\end{array}$ & 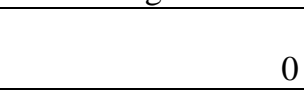 \\
\hline 245 & & 1 & VALVE_PORT $=\langle$ int $\rangle[\mathrm{CCW}]$ & RVLarge \\
\hline 246 & & 1 & $\begin{array}{l}\text { POSITION <float> [IMM or } \\
\text { SYNC] }\end{array}$ & 5000 \\
\hline 247 & & 1 & VALVE_PORT $=\langle$ int $\rangle[\mathrm{CCW}]$ & WasteLarge \\
\hline 248 & & 1 & $\begin{array}{l}\text { POSITION <float> [IMM or } \\
\text { SYNC] }\end{array}$ & 0 \\
\hline 249 & & & LOOP <int $>$ & 2 \\
\hline 250 & & & & \\
\hline 251 & RecleanLWCC & 1 & VALVE_PORT $=\langle$ int $\rangle[\mathrm{CCW}]$ & dILarge \\
\hline 252 & & 1 & $\begin{array}{l}\text { POSITION <float> [IMM or } \\
\text { SYNC] }\end{array}$ & 5000 \\
\hline 253 & & 1 & VALVE_PORT $=\langle$ int $>[\mathrm{CCW}]$ & LWCC \\
\hline 254 & & 1 & $\begin{array}{l}\text { POSITION <float> [IMM or } \\
\text { SYNC] }\end{array}$ & 0 \\
\hline \multicolumn{5}{|l|}{255} \\
\hline 256 & & & LOOP <int $>$ & 14 \\
\hline
\end{tabular}

